\title{
Bilinear Form and Two Bäcklund Transformations for the (3+1)-Dimensional Jimbo-Miwa Equation
}

\author{
He $\mathrm{Li}^{1,2}$ and Yi-Tian Gao ${ }^{1}$ \\ ${ }^{1}$ Ministry-of-Education Key Laboratory of Fluid Mechanics and National Laboratory for Computational Fluid Dynamics, \\ Beijing University of Aeronautics and Astronautics, Beijing 100191, China \\ ${ }^{2}$ State Key Laboratory of Information Photonics and Optical Communications and School of Science, \\ Beijing University of Posts and Telecommunications, Beijing 100876, China \\ Correspondence should be addressed to He Li; lihe771008@163.com
}

Received 27 February 2015; Accepted 12 May 2015

Academic Editor: Luiz Duarte

Copyright (c) $2015 \mathrm{H}$. Li and Y.-T. Gao. This is an open access article distributed under the Creative Commons Attribution License, which permits unrestricted use, distribution, and reproduction in any medium, provided the original work is properly cited.

With Bell polynomials and symbolic computation, this paper investigates the (3+1)-dimensional Jimbo-Miwa equation, which is one of the equations in the Kadomtsev-Petviashvili hierarchy of integrable systems. We derive a bilinear form and construct a bilinear Bäcklund transformation (BT) for the $(3+1)$-dimensional Jimbo-Miwa equation, by virtue of which the soliton solutions are obtained. Bell-polynomial-typed BT is also constructed and cast into the bilinear BT.

\section{Introduction}

Dynamical systems, such as those for the shallow waters [1,2], plasmas and optical fiber communications [3-6], can often be described by the nonlinear evolution equations (NLEEs) [79] and studied by the relevant methods including the inverse scattering [1], Bäcklund transformation (BT) [10-13], and Hirota method [14-16]. Among them, the Hirota method [17, 18 ] is a direct tool for dealing with certain NLEEs and relevant soliton problems $[19,20]$. Based on the bilinear form of a given NLEE, one can obtain the multisoliton solutions [21], bilinear auto-BTs [18], nonlinear superposition formulas, Lax pair, Wronskian formulation [22], and so on [23].

Reflecting the complex nonlinear phenomena in our real world [24-26], higher-dimensional NLEEs with their analytic solutions and integrable properties [27-29] have been of great interest. In fact, some $(2+1)$-dimensional NLEEs have been investigated with different methods, for example, the $(2+1)$-dimensional breaking soliton equation, Kadomtsev-Petviashvili equation, and $(2+1)$-dimensional Kaup-Kupershmidt equation [30-32]. However, for some (3+ 1)-dimensional NLEEs, the conventional integrability test fails [27], and then a natural problem is whether or not there exists BT for a given $(3+1)$-dimensional NLEE. Moreover, for the higher-dimensional NLEEs, finding a bilinear BT via the exchange formula is often difficult, even if possible [18, 21].

In this paper, we will study the following $(3+1)$ dimensional Jimbo-Miwa (JM) equation [32]:

$$
u_{x x x y}+3 u_{y} u_{x x}+3 u_{x} u_{x y}+2 u_{y t}-3 u_{x z}=0,
$$

where $u$ is a real scalar function with four independent variables $x, y, z$, and $t$ and the subscripts denote the corresponding partial derivatives. Seen as one of the equations in the Kadomtsev-Petviashvili hierarchy of integrable systems $[32,33]$, (1) describes certain $(3+1)$-dimensional waves [13, 32] but does not have the Painlevé property [34] as defined in [35]. The soliton [36, 37], periodic [15], rational, and dromion solutions $[38,39]$ for (1) have been obtained. BTs and analytic solitonic solutions have been given in [13] with the truncated Painlevé expansion at the constant level term.

However, existing literature has not studied the bilinear BT and Bell-polynomial-typed BT of (1) as yet. Therefore, in this paper, by means of the Bell polynomials and Hirota bilinear method, we will obtain two BTs for (1), which are different from those in [13]. In Section 2, we will introduce some concepts on the Bell polynomials and their connection with the bilinear forms. In Section 3, using the Bell-polynomial 
expressions, we will derive a bilinear form of (1). In Section 4, based on this bilinear form, we will obtain a bilinear BT with soliton solutions and a Bell-polynomial-typed BT. Finally, our conclusions will be given in Section 5 .

\section{Preliminaries}

Suppose that $\varphi$ is $C^{\infty}$-function with respect to $x$, and $\operatorname{set} \varphi_{\theta x}=$ $\partial_{x}^{\theta} \varphi(\theta=0,1,2, \ldots)$. Then the Bell exponential polynomials are given as [40-43]

$$
Y_{n x}(\varphi) \equiv Y_{n}\left(\varphi_{1 x}, \varphi_{2 x}, \ldots, \varphi_{n x}\right)=e^{-\varphi} \partial_{x}^{n} e^{\varphi},
$$

where $n=1,2, \ldots$

For example,

$$
\begin{aligned}
& Y_{1 x}=\varphi_{1 x}, \\
& Y_{2 x}=\varphi_{2 x}+\varphi_{1 x}^{2}, \\
& Y_{3 x}=\varphi_{3 x}+3 \varphi_{1 x} \varphi_{2 x}+\varphi_{1 x}^{3}, \ldots
\end{aligned}
$$

Two-dimensional Bell polynomials are expressed as [40-43]

$$
\begin{aligned}
& Y_{m x, n t}(\varphi) \equiv Y_{m, n}\left(\varphi_{1 x, 0 t}, \varphi_{0 x, 1 t}, \ldots, \varphi_{r x, s t}, \ldots, \varphi_{m x, n t}\right)=e^{-\varphi} \partial_{x}^{m} \partial_{t}^{n} e^{\varphi}, \\
& \varphi_{r x, s t}=\partial_{x}^{r} \partial_{t}^{s} \varphi(x, t),(m=1,2, \ldots ; r=0,1, \ldots, m ; s=0,1, \ldots, n)
\end{aligned}
$$

with $\varphi$ hereby being $C^{\infty}$-function of $x$ and $t$.

Based on the Bell polynomials given above, the binary Bell polynomials, namely, $\mathcal{Y}$-polynomials, can be defined as [41]

$$
\begin{aligned}
& \mathcal{Y}_{m x, n t}(\nu, w) \equiv Y_{m x, n t}[\varphi(\nu, w)]=Y_{m, n}\left(\varphi_{1 x, 0 t}, \varphi_{0 x, 1 t},\right.
\end{aligned}
$$

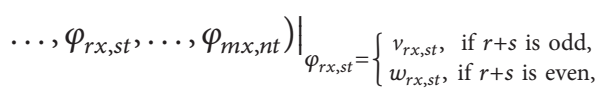

where the vertical line means that the elements on the lefthand side are chosen according to the rule on the righthand side, while $v$ and $w$ are the functions that replace $\varphi$ in the corresponding positions of the Bell polynomials. For simplicity, we denote $\mathscr{Y}_{m x, n t}(v, w)$ as $\mathscr{Y}_{m x}(v, w)$ or $\mathscr{Y}_{n t}(v, w)$ if $n=0$ or $m=0$, respectively.

As one special kind of $\mathscr{Y}$-polynomials, $P$-polynomials only possess the even-order partial differential terms and, with $q=w-v$, are defined as $[40,41]$

$$
\begin{aligned}
& P_{m x, n t}(q) \equiv Y_{m x, n t}[\varphi(0, q)]=Y_{m, n}\left(\varphi_{1 x, 0 t}, \varphi_{0 x, 1 t}, \ldots,\right. \\
& \left.\varphi_{r x, s t}, \ldots, \varphi_{m x, n t}\right)\left.\right|_{\varphi_{r x, s t}}=\left\{\begin{array}{cc}
0, & \text { if } r+s \text { is odd, } \\
q_{r x, s t}, & \text { f } r+s \text { is even, }
\end{array}\right.
\end{aligned}
$$

which vanish unless $n+m$ is even.

According to the above, the lower-order $P$-polynomials can be given as

$$
\begin{aligned}
P_{0}(q) & =1, \\
P_{2 x}(q) & =q_{2 x}, \\
P_{x, t}(q) & =q_{x t}, \\
P_{4 x}(q) & =q_{4 x}+3 q_{2 x}^{2}, \ldots
\end{aligned}
$$

For a given pair of exponentials,

$$
\begin{aligned}
& F=\exp f(x, t), \\
& G=\exp g(x, t),
\end{aligned}
$$

where $f$ and $g$ are $C^{\infty}$-functions of $x$ and $t$, while the Hirota $D$-operators are defined as $[17,42,43]$

$$
\begin{aligned}
& D_{x}^{m} D_{t}^{n} F \cdot G \equiv\left(\frac{\partial}{\partial x}-\frac{\partial}{\partial x^{\prime}}\right)^{m}\left(\frac{\partial}{\partial t}-\frac{\partial}{\partial t^{\prime}}\right)^{n} F(x, t) \\
& \quad \times\left. G\left(x^{\prime}, t^{\prime}\right)\right|_{x^{\prime}=x, t^{\prime}=t},
\end{aligned}
$$

where $x^{\prime}, t^{\prime}$ are the formal variables.

It has been found that there exist some relations between the binary Bell polynomials and the Hirota $D$-operators [40, 41]. When $v=\ln (F / G), w=\ln F G$, a binary Bell polynomial can be transformed into a bilinear term according to the identity $[40,41]$

$$
(F G)^{-1} D_{x}^{n} D_{t}^{m} F \cdot G=\mathscr{Y}_{n x, m t}\left(v=\ln \frac{F}{G}, w=\ln F G\right) .
$$

Likewise, when $w=2 \ln G, P$-polynomials can be associated with the Hirota $D$-operators according to the identity $[40,41]$

$$
\begin{aligned}
(G)^{-2} D_{x}^{n} D_{t}^{m} G \cdot G & =\mathscr{Y}_{n x, m t}(v=0, w=2 \ln G) \\
& =P_{m x, n t}(q) .
\end{aligned}
$$

\section{Bilinear Form}

We will next investigate (1), to be written in $P$-polynomial form with one independent variable. Based on the relation between the binary Bell polynomials and Hirota bilinear operators, namely, identities (10) and (11), (1) can be translated into the corresponding bilinear forms.

Consider the following scale transformations:

$$
\begin{aligned}
& x \longrightarrow \lambda^{k} x^{\prime}, \\
& y \longrightarrow \lambda^{l} y^{\prime}, \\
& z \longrightarrow \lambda^{\alpha} z^{\prime}, \\
& t \longrightarrow \lambda^{\beta} t^{\prime}, \\
& u \longrightarrow \lambda^{\mu} u^{\prime},
\end{aligned}
$$

where $\lambda, k, l, \alpha, \beta$, and $\mu$ are the real constants. Invariance of (1) under such transformations requires that $\mu=-k, \alpha=$ $l+2 k$, and $\beta=3 k$.

Notice that if we require that $\mu=-k$, we have to set $u=$ $c q_{x}$ in (1) and obtain

$$
q_{4 x, y}+3 c q_{x y} q_{3 x}+3 c q_{2 x} q_{2 x, y}+2 q_{x y t}-3 q_{2 x, z}=0,
$$

where $c$ is an arbitrary constant. 
In order to express (13) with $P$-polynomials, we choose $c=1$. Then

$$
\left[P_{3 x, y}(q)+2 P_{y t}(q)-3 P_{x, z}(q)\right]_{x}=0,
$$

whose corresponding bilinear form is

$$
\left(D_{x}^{3} D_{y}+2 D_{y} D_{t}-3 D_{x} D_{z}\right) G \cdot G=0 .
$$

Therefore, we get the bilinear form of (1), which is (14) with $P$-polynomials or (15) with the bilinear operators. We note that (15) is the same as that in [15], but the method that we used is different from that in [15].

\section{Bell-Polynomial-Typed BT and Bilinear BT with Soliton Solutions}

To construct a BT, we express (1) with $P$-polynomials:

$$
E(q)=P_{3 x, y}(q)+2 P_{y t}(q)-3 P_{x, z}(q) .
$$

Based on $E(q)$ we will derive the Bell-polynomial-typed BT under the homogenous constraints between the primary and replica fields instead of using exchange formulae.

Using the Bell polynomials, we have

$$
\begin{aligned}
& c(v, w)=E\left(q^{\prime}\right)-\left.E(q)\right|_{q=2 \ln G, q^{\prime}=2 \ln F}=E(w+v) \\
& \quad-\left.E(w-v)\right|_{w=\ln F G, v=\ln (F / G)}=(w+v)_{3 x, y} \\
& +3(w+v)_{x y}(w+v)_{2 x}+2(w+v)_{y t}-3(w+v)_{x z} \\
& \quad-\left[(w-v)_{3 x, y}+3(w-v)_{x y}(w-v)_{2 x}\right. \\
& \left.+2(w-v)_{y t}-3(w-v)_{x z}\right]=2 v_{3 x, y}+6 w_{x y} v_{x x} \\
& +6 v_{x y} w_{x x}+4 v_{y t}-6 v_{x z} .
\end{aligned}
$$

Note that

$$
\begin{aligned}
\mathscr{Y}_{2 x, y} & =v_{2 x, y}+v_{y} w_{2 x}+2 v_{x} w_{x y}+v_{y} v_{x}^{2}, \\
\mathscr{Y}_{3 x} & =v_{3 x}+3 v_{x} w_{2 x}+v_{x}^{3} .
\end{aligned}
$$

Therefore, substituting (18) into (17), we have

$$
\begin{aligned}
\frac{c(v, w)}{2}= & v_{3 x, y}+3 w_{x y} v_{x x}+3 v_{x y} w_{x x}+2 v_{y t}-3 v_{x z} \\
= & {\left[a v_{3 x, y}+(1-a) v_{3 x, y}\right]+2 v_{y t}-3 v_{x z} } \\
& +3 w_{x y} v_{2 x}+3 v_{x y} w_{2 x} \\
= & \left(a \mathscr{Y}_{2 x, y}\right)_{x}-a\left(v_{y} w_{2 x}+2 v_{x} w_{x y}+v_{y} v_{x}^{2}\right)_{x} \\
& +(1-a)\left(\mathscr{Y}_{3 x}\right)_{y} \\
& -(1-a)\left(3 v_{x} w_{2 x}+v_{x}^{3}\right)_{y}+2\left(\mathscr{Y}_{t}\right)_{y} \\
& -3\left(\mathscr{Y}_{z}\right)_{x}+3 w_{x y} v_{2 x}+3 v_{x y} w_{2 x} \\
= & \left(a \mathscr{Y}_{2 x, y}-3 \mathscr{Y}_{z}\right)_{x}+\left[(1-a) \mathscr{Y}_{3 x}+2 \mathscr{Y}_{t}\right]_{y} \\
& +A,
\end{aligned}
$$

$$
\begin{aligned}
A= & 3 w_{x y} v_{2 x}+3 v_{x y} w_{2 x} \\
& -a\left(v_{y} w_{2 x}+2 v_{x} w_{x y}+v_{y} v_{x}^{2}\right)_{x} \\
& -(1-a)\left(3 v_{x} w_{2 x}+v_{x}^{3}\right)_{y},
\end{aligned}
$$

and $a$ is an arbitrary constant.

Further computation shows that

$$
\begin{aligned}
A= & 2 a v_{x y} w_{2 x}+(a-3) v_{x} w_{2 x, y}+(2 a-3) v_{x}^{2} v_{x y} \\
& -(2 a-3) v_{2 x} w_{x y}-a v_{y} w_{3 x}-2 a v_{x} v_{y} v_{2 x} \\
= & 2 a\left(v_{x} \mathscr{Y}_{2 x}\right)_{y}+\left(-2 a-\frac{3}{2}\right) v_{x}\left(\mathscr{Y}_{2 x}\right)_{y} \\
& -a v_{y}\left(\mathcal{Y}_{2 x}\right)_{x} \\
& +\left[\left(a-\frac{3}{2}\right) v_{x} w_{2 x, y}+(-2 a+3) v_{2 x} w_{x y}\right] .
\end{aligned}
$$

Hereby, if we choose $a=3 / 2$ and set $\mathscr{Y}_{2 x}=\sigma$, then

$$
\begin{aligned}
A= & 2 a\left(v_{x} \mathscr{Y}_{2 x}\right)_{y}=3 \tau v_{x y}, \\
c(v, w) & \\
= & 2\left\{\left(a \mathscr{Y}_{2 x, y}-3 \mathscr{Y}_{z}\right)_{x}+\left[(1-a) \mathscr{Y}_{3 x}+2 \mathscr{Y}_{t}\right]_{y}\right\} \\
& +6 \sigma v_{x y} .
\end{aligned}
$$

Hence,

$$
\begin{aligned}
\frac{c(v, w)}{2}= & {\left[\frac{3}{2} \mathscr{Y}_{2 x, y}-3 \mathscr{Y}_{z}+(3-b) \sigma \mathscr{Y}_{y}\right]_{x} } \\
& +\left(-\frac{1}{2} \mathscr{Y}_{3 x}+2 \mathscr{Y}_{t}+b \sigma \mathscr{Y}_{x}\right)_{y} .
\end{aligned}
$$

Moreover, a decomposition of (23) leads to the following Bellpolynomial-typed BT:

$$
\begin{aligned}
\mathscr{Y}_{2 x} & =\sigma, \\
\frac{3}{2} \mathscr{Y}_{2 x, y}-3 y_{z}+(3-b) \sigma y_{y} & =\tau, \\
-\frac{1}{2} y_{3 x}+2 y_{t}+b \sigma y_{x} & =\delta,
\end{aligned}
$$

where $b, \sigma, \tau$, and $\delta$ are the arbitrary constants.

Using the connection between the Bell polynomials and bilinear operators, we give a bilinear BT between $G$ and $G^{\prime}$ as

$$
\begin{aligned}
D_{x}^{2} G^{\prime} \cdot G & =\sigma G^{\prime} \cdot G \\
{\left[\frac{3}{2} D_{x}^{2} D_{y}-3 D_{z}+(3-b) \sigma D_{y}\right] G^{\prime} \cdot G } & =\tau G^{\prime} \cdot G, \\
\left(-\frac{1}{2} D_{x}^{3}+2 D_{t}+b \sigma D_{x}\right) G^{\prime} \cdot G & =\delta G^{\prime} \cdot G .
\end{aligned}
$$


As an application, we derive the one-soliton solutions from a trivial solution, by virtue of the bilinear BT, that is, (25). Taking $\tau=0, \delta=0$, and $G^{\prime}=1$ in (25), we get

$$
\begin{aligned}
G_{x x} & =\sigma G, \\
\frac{3}{2} G_{x x y}-3 G_{z}+(3-b) \sigma G_{y} & =0, \\
-\frac{1}{2} G_{3 x}+2 G_{t}+b \sigma G_{x} & =0 .
\end{aligned}
$$

Substituting (26) into (28), we have

$$
\left(b-\frac{1}{2}\right) G_{3 x}+2 G_{t}=0,
$$

and hence we take

$$
G=e^{\xi}+e^{-\xi}
$$

where $\xi=k x+l y+m z+\omega t+\xi^{(0)}$ and $\xi^{(0)}$ is a nonzero constant. On the other hand, we can choose $\sigma=k^{2}$ and substitute it into (26) and (29), which implies that

$$
\omega=\left(\frac{1}{4}-\frac{b}{2}\right) k^{3} .
$$

Similarly, substituting (26) into (27) leads to

$$
\left(\frac{9}{2}-b\right) k^{2} G_{y}=3 G_{z} .
$$

Solving (32), we obtain

$$
m=\left(\frac{3}{2}-\frac{b}{3}\right) k^{2} l .
$$

Finally, we can present the one-soliton solutions of (1) as

$$
u=2(\ln G)_{x}=2 k \tanh \xi
$$

where $\xi=k x+l y+(3 / 2-b / 3) k^{2} l z+(1 / 4-b / 2) k^{3} t+\xi^{(0)}$, while the parameters $k, l, b$, and $\xi^{(0)}$ are all arbitrary constants.

\section{Discussions and Conclusions}

We have investigated the $(3+1)$-dimensional Jimbo-Miwa equation, that is, (1). With the aid of the Bell polynomials and Hirota bilinear operators, we have derived bilinear form (16) of (1) and then constructed a new BT, that is, (25), with the Bell polynomials and symbolic computation. The bilinear form and BT are important integrable property for the nonlinear evolution equations. Moreover, a BT often can be cast into the Lax pair for integrable equations. It may be possible to construct the bilinear BTs for the $(3+1)$-dimensional Jimbo-Miwa equation via the exchange formulae; however, the computation is tedious. Bell-polynomial-typed BTs (24) have been constructed hereby and then cast into bilinear BTs (25), which help us avoid the difficulties in using the exchange formulae. As an application, one-soliton solutions (34) have been obtained via BT (25). The existence of solution obtained via solving this BT indicates that (24) or (25) are genuine ones.

\section{Conflict of Interests}

The authors declare that there is no conflict of interests regarding the publication of this paper.

\section{Acknowledgments}

The authors would like to express their sincere thanks to Mr. Q. X. Qu and Mr. K. Sun for their helpful suggestions. This work has been supported by the National Natural Science Foundation of China under Grant no. 11272023, by the Open Fund of State Key Laboratory of Information Photonics and Optical Communications (Beijing University of Posts and Telecommunications), and by the Fundamental Research Funds for the Central Universities of China under Grant no. 2011BUPTYB02.

\section{References}

[1] M. J. Ablowitz and P. A. Clarkson, Solitons, Nonlinear Evolution Equations and Inverse Scattering, Cambridge University Press, Cambridge, UK, 1991.

[2] M. P. Barnett, J. F. Capitani, J. von zur Gathen, and J. Gerhard, "Symbolic calculation in chemistry: selected examples," International Journal of Quantum Chemistry, vol. 100, no. 2, pp. 80-104, 2004.

[3] X. Lü, H.-W. Zhu, X.-H. Meng, Z.-C. Yang, and B. Tian, “Soliton solutions and a Bäcklund transformation for a generalized nonlinear Schrödinger equation with variable coefficients from optical fiber communications," Journal of Mathematical Analysis and Applications, vol. 336, no. 2, pp. 1305-1315, 2007.

[4] X. Lü, "Bright-soliton collisions with shape change by intensity redistribution for the coupled Sasa-Satsuma system in the optical fiber communications," Communications in Nonlinear Science and Numerical Simulation, vol. 19, no. 11, pp. 3969-3987, 2014.

[5] B. Tian, Y. T. Gao, and H. W. Zhu, "Variable-coefficient higherorder nonlinear Schrödinger model in optical fibers: variablecoefficient bilinear form, Bäcklund transformation, brightons and symbolic computation," Physics Letters A, vol. 366, pp. 223229, 2007.

[6] B. Tian and Y. T. Gao, "Symbolic computation on cylindricalmodified dust-ion-acoustic nebulons in dusty plasmas," Physics Letters A, vol. 362, no. 4, pp. 283-288, 2007.

[7] Z.-Y. Yan and H.-q. Zhang, "Symbolic computation and new families of exact soliton-like solutions to the integrable BroerKaup (BK) equations in $(2+1)$-dimensional spaces," Journal of Physics A: Mathematical and General, vol. 34, no. 8, pp. 17851792, 2001.

[8] X. Lü and M. Peng, "Systematic construction of infinitely many conservation laws for certain nonlinear evolution equations in mathematical physics," Communications in Nonlinear Science and Numerical Simulation, vol. 18, no. 9, pp. 2304-2312, 2013.

[9] X. Lü and M. Peng, "Nonautonomous motion study on accelerated and decelerated solitons for the variable-coefficient Lenells-Fokas model," Chaos, vol. 23, no. 1, Article ID 013122, 2013.

[10] C. Rogers and W. F. Shadwick, Bäacklund Transformations and Their Applications, Academic Press, New York, NY, USA, 1982. 
[11] X. Lü, "New bilinear Bäcklund transformation with multisoliton solutions for the (2+1)-dimensional Sawada-Kotera model," Nonlinear Dynamics, vol. 76, no. 1, pp. 161-168, 2014.

[12] X. Lü and J. Li, "Integrability with symbolic computation on the Bogoyavlensky-Konoplechenko model: BELl-polynomial manipulation, bilinear representation, and Wronskian solution," Nonlinear Dynamics, vol. 77, no. 1-2, pp. 135-143, 2014.

[13] B. Tian, Y.-T. Gao, and W. Hong, "The solitonic features of a nonintegrable $(3+1)$-dimensional Jimbo-Miwa equation," Computers \& Mathematics with Applications, vol. 44, no. 3-4, pp. 525-528, 2002.

[14] J. F. Zhang and F. M. Wu, "Bäcklund transformation and multiple soliton solutions for the $(3+1)$-dimensional JimboMiwa equation," Chinese Physics, vol. 11, no. 5, p. 425, 2002.

[15] Q. L. Zha and Z. B. Li, "Multiple periodic-soliton solutions for (3+1)-dimensional Jimbo-Miwa equation," Communications in Theoretical Physics, vol. 50, no. 5, pp. 1036-1040, 2008.

[16] X. Lü, "Soliton behavior for a generalized mixed nonlinear Schrödinger model with $N$-fold Darboux transformation," Chaos, vol. 23, Article ID 033137, 2013.

[17] R. Hirota, "Exact solution of the korteweg_de Vries equation for multiple collisions of solitons," Physical Review Letters, vol. 27, no. 18, pp. 1192-1194, 1971.

[18] R. Hirota, "A new form of Bäcklund transformations and its relation to the inverse scattering problem," Progress of Theoretical Physics, vol. 52, no. 5, pp. 1498-1512, 1974.

[19] R. Hirota and J. Satsuma, "A variety of nonlinear network equations generated from the bäcklund transformation for the toda lattice," Progress of Theoretical Physics Supplements, vol. 59, pp. 64-100, 1976.

[20] R. Hirota, X.-B. Hu, and X.-Y. Tang, "A vector potential KdV equation and vector Ito equation: soliton solutions, bilinear Bäcklund transformations and Lax pairs," Journal of Mathematical Analysis and Applications, vol. 288, no. 1, pp. 326-348, 2003.

[21] R. Hirota, The Direct Method in Soliton Theory, Cambridge University Press, Cambridge, UK, 2004.

[22] Y. Matsuno, Bilinear Transformation Method, vol. 174 of Mathematics in Science and Engineering, Academic Press, London, UK, 1984.

[23] C.-X. Li, W.-X. Ma, X.-J. Liu, and Y.-B. Zeng, "Wronskian solutions of the Boussinesq equation-solitons, negatons, positons and complexitons," Inverse Problems, vol. 23, no. 1, pp. 279-296, 2007.

[24] H. Aratyn, L. A. Ferreira, and A. H. Zimerman, "Exact static soliton solutions of (3+1)-dimensional integrable theory with nonzero Hopf numbers," Physical Review Letters, vol. 83, no. 9, pp. 1723-1726, 1999.

[25] A. Wazwaz, "Integrable (2+1)-dimensional and (3+1)-dimensional breaking soliton equations," Physica Scripta, vol. 81, no. 3, Article ID 035005, 2010.

[26] Y. T. Gao and B. Tian, "New family of overturning soliton solutions for a typical breaking soliton equation," Computers \& Mathematics with Applications, vol. 30, no. 12, pp. 97-100, 1995.

[27] J. Yu and Z. Lou, "A $(3+1)$-dimensional Painlevé integrable model obtained by deformation," Mathematical Methods in the Applied Sciences, vol. 25, no. 2, pp. 141-148, 2002.

[28] J. B. Chen, "Finite-gap solutions of $2+1$ dimensional integrable nonlinear evolution equations generated by the Neumann systems," Journal of Mathematical Physics, vol. 51, no. 8, Article ID 083514, 2010.
[29] R. S. Ward, "Nontrivial scattering of localized solitons in a (2+1)dimensional integrable system," Physics Letters A, vol. 208, no. 3, pp. 203-208, 1995.

[30] J. F. Zhang, "Multiple soliton-like solutions for $(2+1)$-dimensional dispersive Long-Wave equations," International Journal of Theoretical Physics, vol. 37, no. 9, pp. 2449-2455, 1998.

[31] A. V. Mikhailov and R. I. Yamilov, "Towards classification of $(2+$ 1)-dimensional integrable equations," Journal of Physics A, vol. 31, Article ID 6707, 1998.

[32] M. Jimbo and T. Miwa, "Solitons and infinite dimensional Lie algebras," Publications of the Research Institute for Mathematical Sciences, vol. 19, pp. 943-1001, 1983.

[33] X.-Y. Tang and J. Lin, "Conditional similarity reductions of Jimbo-Miwa equations via the classical Lie group approach," Communications in Theoretical Physics, vol. 39, no. 1, pp. 6-8, 2003.

[34] B. Dorrizzi, B. Grammaticos, A. Ramani, and P. Winternitz, "Are all the equations of the KP hierarchy integrable?" Journal of Mathematical Physics, vol. 27, pp. 2848-2852, 1986.

[35] J. Weiss, M. Tabor, and G. Carnevale, “The Painlevé property for partial differential equations," Journal of Mathematical Physics, vol. 24, no. 3, pp. 522-526, 1983.

[36] C.-L. Bai and H. Zhao, "Some special types of solitary wave solutions for $(3+1)$-dimensional Jimbo-Miwa equation," Communications in Theoretical Physics, vol. 41, no. 6, pp. 875-877, 2004.

[37] A.-M. Wazwaz, "Multiple-soliton solutions for the CalogeroBogoyavlenskii-Schiff, Jimbo-Miwa and YTSF equations," Applied Mathematics and Computation, vol. 203, no. 2, pp. 592-597, 2008.

[38] G. Q. Xu, “The soliton solutions, dromions of the KadomtsevPetviashvili and Jimbo-Miwa equations in $(3+1)$-dimensions," Chaos, Solitons and Fractals, vol. 30, no. 1, pp. 71-76, 2006.

[39] S.-H. Ma, J.-P. Fang, B.-H. Hong, and C.-L. Zheng, "New exact solutions and interactions between two solitary waves for (3+1)-dimensional jimbo-miwa system," Communications in Theoretical Physics, vol. 49, no. 5, pp. 1245-1248, 2008.

[40] C. Gilson, F. Lambert, J. J. Nimmo, and R. Willox, "On the combinatorics of the Hirota D-operators," Proceedings of the Royal Society of London A: Mathematical, Physical and Engineering Sciences, vol. 452, no. 1945, pp. 223-234, 1996.

[41] F. Lambert and J. Springael, "On a direct procedure for the disclosure of Lax pairs and Bäcklund transformations," Chaos, Solitons \& Fractals, vol. 12, no. 14-15, pp. 2821-2832, 2001.

[42] X. Lü, B. Tian, K. Sun, and P. Wang, "Bell-polynomial manipulations on the Bäcklund transformations and Lax pairs for some soliton equations with one tau-function," Journal of Mathematical Physics, vol. 51, no. 11, Article ID 113506, 2010.

[43] X. Lü, F. H. Lin, and F. H. Qi, "Analytical study on a twodimensional Korteweg-de Vries model with bilinear representation, Bäcklund transformation and soliton solutions," Applied Mathematical Modelling, vol. 39, pp. 3221-3226, 2015. 


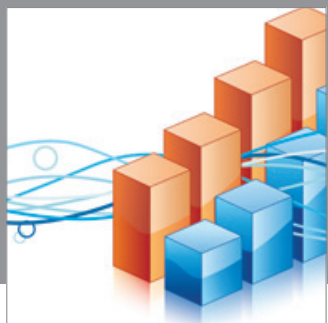

Advances in

Operations Research

mansans

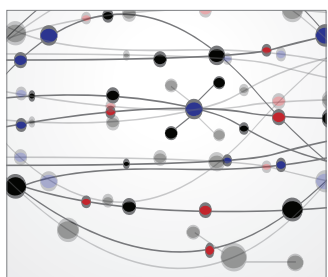

The Scientific World Journal
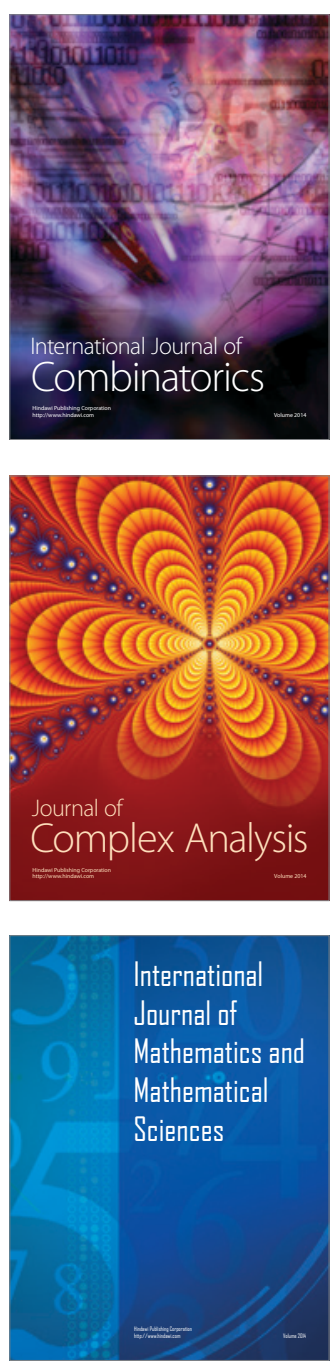
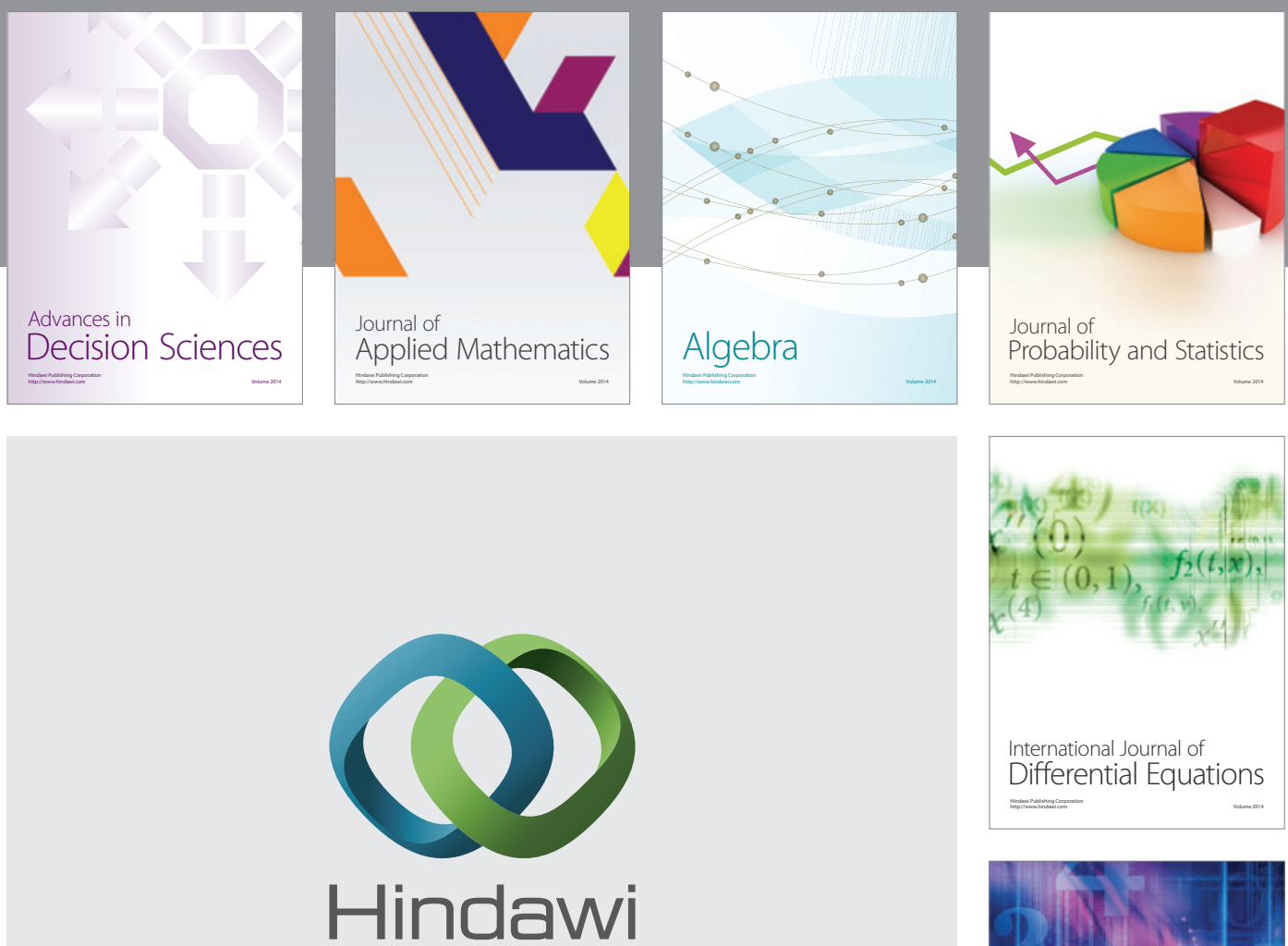

Submit your manuscripts at http://www.hindawi.com
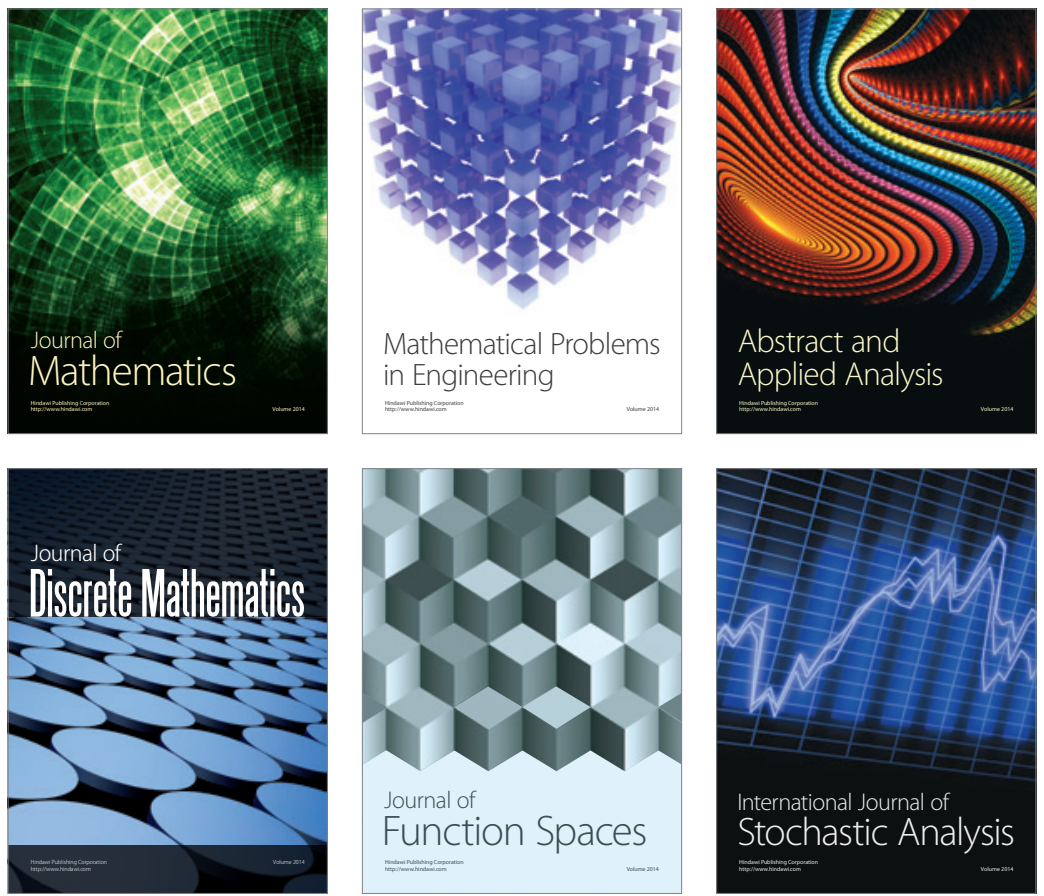

Journal of

Function Spaces

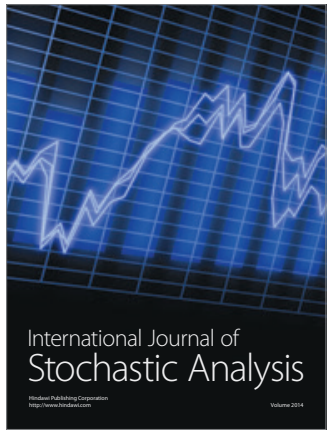

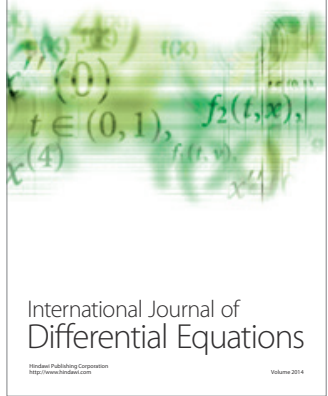
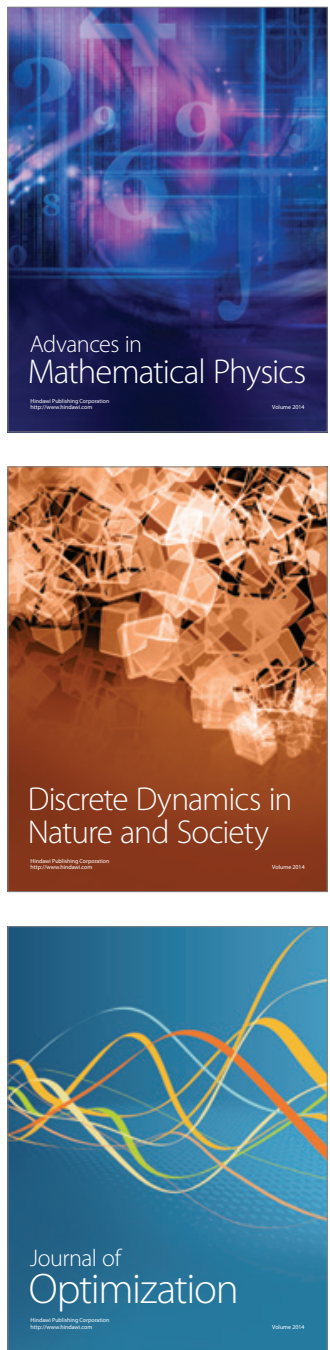